\title{
Optically pumped mirrorless lasing. A Review. Part II. Lasing in photonic crystals and microcavities
}

\author{
Dudok T. H. and Nastishin Yu. A.
}

Institute of Physical Optics, 23 Dragomanov Street, 79005 Lviv, Ukraine

Received: 31.01 .2014

\begin{abstract}
This article is a second part of the review on optically pumped mirrorless lasing. Consideration of random lasing presented in the first part [Nastishin $\mathrm{Yu} \mathrm{A}$ and Dudok T H, 2013. Ukr. J. Phys. Opt.] is now followed by analysis of the literature on the lasing in photonic structures, which is mainly focussed on dye-doped cholesteric liquid crystals and microcavities, including liquid-crystal microdroplets.
\end{abstract}

Keywords: mirrorless lasing, mirrorless lasers, photonic bandgap edge lasing, dye-doped cholesteric liquid crystals, liquid crystal lasers, whispering-gallery modes, optical microcavities

PACS: 42.55.Mv, 42.55.Sa, 42.55.Tv, 42.70.Qs, 61.30.-v, 77.84.Nh, 78.60.Lc

UDC: $535.37+535.35+681.7 .069 .24+52-626$

\section{Introduction}

Laser generation defined as a stimulated light emission amplified in a resonant cavity sensu stricto is a Purcell effect [1], which sensu lato implies modification of the emission rate of electromagnetic radiation by its coupling to resonator modes. The resonator provides a feedback necessary for resonant amplification. The Purcell effect which is at the heart of feedback for all of the lasing mechanisms known up to date can be realized in a number of ways. The common requirement is coupling of the emission spectrum to at least one mode of the resonator. Then the resonator selects the emission at the frequency coinciding with the resonant mode(s) and amplifies it(them) by a factor $f=3 Q(\lambda / n)^{3} /\left(4 \pi^{2} V\right)$, which is known as a Purcell factor. Here $\lambda$ denotes the emission wavelength in vacuum and $n$ the refractive index of the resonator.

A posteriori it is clear that the lasing efficiency is governed by the two main parameters: the quality factor $Q$ of the resonator and the mode volume $V$. The working principle for all commercially available light resonators is multiple light reflection and, consequently, the $Q$ parameter is governed by the mirror reflection coefficient. Modern supermirrors with extremely low losses provide ultra-high $Q$-factors of the order of $10^{11}$. However, due to technological difficulties, their fabrication and optical alignment have become a bottleneck of laser production. When the technology reaches its saturation, the closest way to advance is to see, if possible, of how the problem is solved in the Nature. Indeed, recently it has become clear that there is a wealth of naturally existing resonators based on different phenomena providing alternative ways for the multiple light reflection. A Bragg diffraction in the photonic structures, total internal reflection in the closed cavities, surface plasmon resonances in the metal nanoparticles, and multiple light scattering in the random media leading to light localization are among such solutions. The random and SPASER-based lasers have been discussed at length in the first part of our review [2]. Here we 
review the literature on the two other mechanisms of the mirrorless lasing, namely on the photonic bandgap $(\mathrm{PhBG})$ edge lasing and the lasing in closed microcavities.

\section{Lasing in photonic crystals}

Periodic optical structures which are now called as photonic crystals (PhCs) have been studied in one or another forms for more than 120 years. Probably, the first publications are those by Stokes [3] in 1885 and by Rayleigh [4] in 1887, who have discovered the phenomenon of selective light reflection (SLR) in layered crystalline structures, which evidently belong to $1 \mathrm{D} \mathrm{PhCs}$. The progress in the optics of periodic structures has been greatly influenced by the needs in explanation of many optical observations in liquid crystals (LCs), some of which spontaneously form photonic structures in the form of thermodynamically stable phases or periodical distortions in the layered LCs [5, 6, 7] and of lasing with the distributed feedback [8-10]. The concept of the photonic structures has been formulated in 1980s by Eli Yablonovitch [11] and Sajeev John [12-14].

It is true that some of the basic concepts associated with the photonic media are not quite new and have been known in frames of the other fields such as the optics of stratified media, which covers the notions of selective Bragg reflection and distributed feedback. The importance of the newly announced concept is that it generalizes the previous knowledge and manifests itself as a powerful tool for predicting novel optical effects. The term $\mathrm{PhC}$ has been coined by Eli Yablonovitch [15]. The photonic media are naturally existent or artificially fabricated structures with the structural organization satisfying conditions of resonances for some optical modes that reveal the SLR. Consequently, the light transmission spectrum of a $\mathrm{PhC}$ contains a spectral region called as PhBG, where some light modes are totally backward-reflected and so turn out to be totally prohibited for transmitting. However, it is worth noticing that the $\mathrm{PhC}$ is not a trivial mirror, simply reflecting light backward; it is rather a medium with the distributed feedback, which implies that the light wave is multiply reflected within the PhC structure, forming a quasi-standing wave with the backward leakage, and showing up as the SLR.

There is a close analogy between the PhCs and semiconductors. As remarked by Dowling et al. [16], formation of a standing light wave near the $\mathrm{PhBG}$ edges in a $\mathrm{PhC}$ corresponds to quasi-standing de Broglie wave in a semiconductor for an electron, whose effective mass becomes very large at the band-edge energies. John and Quang [17] have predicted an enhancement of nonlinear phenomena, including superradiance at the edge of the PhBG in a 3D PhC. They claim that, while the maximum gain of the dye atoms is at the wavelength nearby the band edge, the superradiant emission becomes localized in the vicinity of the atoms. In 1994 Dowling et al. [16] have shown that the photon group velocity approaches zero near the edge of a 1D photonic bandgap. The latter is a result of multiple light reflections within the photonic structure and implies that the photons with the wavelength close to the edge of $\mathrm{PhBG}$ spend in the structure much longer time than the other photons. Therefore, the optical path length for the photons of the proper wavelength becomes anomalously long. Slow light near the $\mathrm{PhBG}$ edges is a general property of the photonic structures [18]. As shown in Ref. [16], if a PhC is a gain material itself or is doped by appropriate dye molecules, then the gain near the photonic band edge can be enhanced by a factor more than four, thus leading to the stop-band-edge lasing. Computer simulations performed in Ref. [16] indeed reveal that the optical field at the $\mathrm{PhBG}$ edges inside a $\mathrm{PhC}$ is a quasi-standing wave.

Significant lowering of the lasing threshold in the $\mathrm{PhCs}$ is a direct consequence of the stop-band localization of photons. In a PhC layer of finite thickness, the group velocity drops gradually to its minimum after not less than about 10 periods, such that the increase of the gain is not 
uniform through the structure but is highest near its middle [16]. The latter also implies that the lasing threshold should be thickness-dependent. Theoretically the stop-band lasing can be observed at any $\mathrm{PhBG}$ edges, although the two light modes should have different thresholds. The experiments on the radiation emission of a point source embedded inside a $\mathrm{PhC}$ confirm that the enhancement of radiation occurs at the PhBG edges $[19,20]$.

Holographic approach has been recently used [21] to pattern a periodic dispersion of the LC inside a polymer matrix. Lasing in holographically patterned dye-doped polymer dispersed LCs (PDLC) films has been suggested in Ref. [22] for multi-dimensional multi-colour display technologies. Lasing from such a holographically patterned dye-doped PDLC films can be electrically switchable [23]. A colour-tunable lasing controlled by variations of the temperature or the electric field applied has been realized in Ref. [24] while embedding a dye-doped cholesteric LC (ChLC) in holographically patterned polymeric microchannels. 3D photonic metamaterials at the optical frequencies have been reported in Ref. [25]. The main drawback of the artificially fabricated $\mathrm{PhCs}$ is that sophisticated technologies are needed to produce them, whereas the photonic LC structures are formed spontaneously and are easy in preparation. In the next section we review the literature on lasing in the LC phases.

\section{Lasing in liquid crystals}

LCs are materials composed of anisometric structural units that manifest long-range orientational ordering at lowered dimensionality $\mathrm{D}$ of the positional ordering. The latter simply means that the material is liquid at least in one spatial direction. In thermotropic calamitic nematics, the rod-like molecules tend to orient their long axes parallel to a common direction called a nematic director $\hat{n}$. Chiral nematics with the director field spontaneously helically twisted around some axis perpendicular to $\hat{n}$ are called cholesterics. On heating a ChLC phase can transform into LC phases called as blue phases (BPs). Another type of helically arranged orientation of the molecules takes place in chiral smectic $C^{*}\left(\mathrm{Sm} C^{*}\right)$ phases, where molecules form equidistant planes (called as smectic layers), such that the long axes of the molecules are tilted with respect to the smectic layers and helically rotate around the layer normal from one layer to another. Due to the SLR property, the cholesteric and $\mathrm{Sm} C^{*}$ phases are 1D PhCs, whereas BPI and BPII are 3D PhCs. Photonic effects in a swollen lamellar $L_{\alpha}$ phase have been studied in Ref. [26].

The helical twist in the LC phases is characterized by the pitch $P$, which for the cholesteric and $\mathrm{BP}$ phases is a distance measured along the helical axis that corresponds to rotation of the director by $\pi$. Notice that due to the property $\hat{n}=-\hat{n}$, the pitch in the cholesterics and BPs corresponds to the rotation by $\pi$ but not $2 \pi$. Due to space periodicity of the director field, the cholesteric structure acts as a Bragg mirror and selectively reflects a circularly polarized light, which is of the same handedness as the cholesteric structure for the light wavelengths $\lambda_{S}$ satisfying the condition $\lambda_{\perp}=P n_{\perp}<\lambda_{S}<P n_{\|}=\lambda_{\|}$. Here $n_{\perp}$ and $n_{\|}$are the refractive indices for the light polarizations respectively perpendicular and parallel to the local director orientation. Because the propagation of one of the two circularly polarized light modes is totally inhibited inside the SLR band, the cholesteric selective reflection band is in fact a PhBG. The group velocity $v_{g}$ for this light mode tends to zero and consequently the density of states of photons, which is inversely proportional to $v_{g}$, diverges while approaching both of the PhBG edges.

When the light propagates along the helical axis, the two eigenwaves are nearly circularly polarized waves, with the opposite handedness far from the PhBG on both its sides. The wave with 
the handedness opposite to that of the cholesteric twist does not 'feel' the PhBG; it propagates with no reflection through the structure and remains circularly polarized, whereas the other wave suffers the SLR within the PhBG and its polarization drastically changes in the vicinity of the PhBG and across it. At the both PhBG edges, the light wave suffering the selective reflection becomes linearly polarized. The directions of the linear polarizations are parallel and perpendicular to the local director respectively at the two $\mathrm{PhBG}$ edges $[27,28]$. For conventional thermotropic nematics with rod-like molecules we have $n_{\|}>n_{\perp}$ and, hence, the light mode is polarized parallel to the local director at the long-wavelength side $\left(\lambda_{\|}=P n_{\|}\right)$of the PhBG and the polarization becomes perpendicular to the local director at the short-wavelength side $\left(\lambda_{\perp}=P n_{\perp}\right)$. The polarization of this mode varies across the PhBG from the linear at one PhBG edge, through elliptical and circular, to the perpendicular linear at the opposite edge. However, to tell the truth, the values $\lambda_{\perp}$ and $\lambda_{\|}$that mark the PhBG edges as defined above are only first approximations of the exact values given by the theory developed by de Vrie (see [27, 28]).

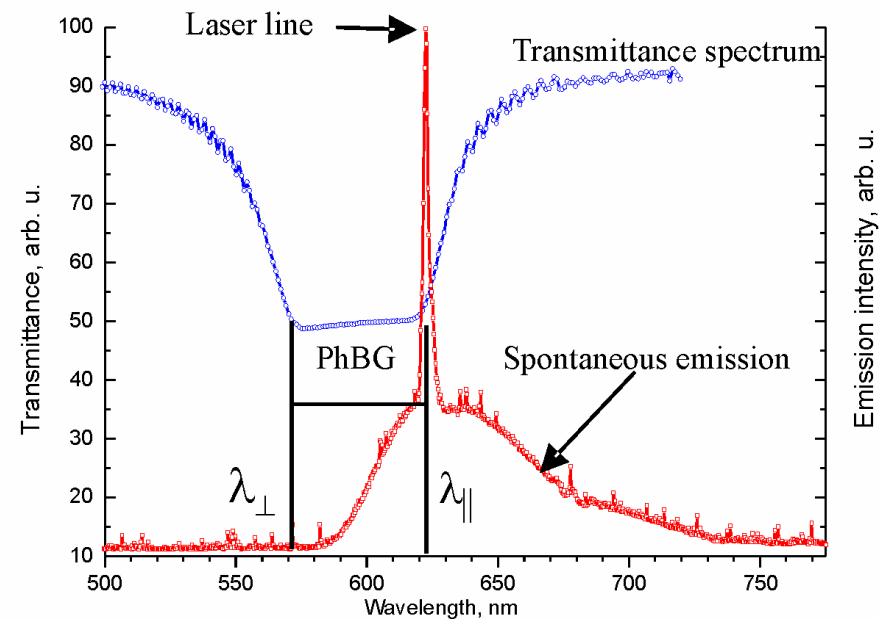

Fig. 1. (Colour online). Emission spectrum (red line) of a cholesteric mixture KET90700+E7(21 wt.\% ) doped with a dye DG-56(1 wt.\%), as measured after optical pumping at $\lambda=532 \mathrm{~nm}$. Thin vertical lines marked by $\lambda_{\perp}$ and $\lambda_{\|}$ show the edges of the cholesteric PhBG observed in the transmission spectrum (blue line). The composition of the cholesteric mixture is chosen so that to match the long-wavelength PhBG edge with the maximum of dye-fluorescence emission band. Laser line coincides with the long-wavelength side $\lambda_{\|}$of the PhBG.

If the ChLC is a gain medium or is doped with an appropriate dye, the stop-band lasing can be observed at the both PhBG edges but their pumping-intensity thresholds are not the same. We have obtained lasing in a cholesteric mixture KET 90700 (from Jiangsu Hecheng Chemical Materials Co., Ltd $(\mathrm{HCCH}))+21$ wt. \% nematic E7 (from Merk) doped with 1 wt \% of a dye DG-56 ((4-((E)-2-( $N$-(2-ethylhexyl)-1,8-naphthalimid-4-yl)vinyl)phenyl)benzenamine). Fig. 1 illustrates a position of the lasing line at the long-wavelength side of the PhBG.

The polarizations of the band-edge laser modes depend on the scalar order parameter $S_{d}$ of orientation of the transition dipole moment $\vec{d}$ of dye molecules in the ChLC structure. As shown in Ref. [28], for an ideal case $S_{d}=1$ describing the perfect orientation $(\vec{d} \| \hat{n})$, the lowest pumping threshold corresponds to the laser mode located at the long-wavelength side $\lambda_{\|}$of the PhBG, while its polarization is expected to be circular, having the same handedness as that of the ChLC helix. If 
the second mode at the short-wavelength side $\lambda_{\perp}$ could be observed at higher pumping intensities, then its polarization would be also circular, though with the handedness opposite to that of the ChLC helix. In the alternative case $S_{d}=-1 / 2$ corresponding to the orientation $\vec{d} \perp \hat{n}$, the lowest pumping threshold corresponds to the laser mode located at the short-wavelength side $\lambda_{\perp}$ of the $\mathrm{PhBG}$ and its polarization is also circular, with the handedness being the same as that of the ChLC helix. If the second laser mode is also observed at $\lambda_{\|}$, then its circular polarization would be of the opposite handedness with respect to that of the ChLC helix. In any real samples $S_{d}$ is far from these extreme values and we have $0 \leq S_{d}<1$ in the most of cases. If $0 \leq S_{d}<1$, the theory [28] shows that the polarization is circular, with the same handedness as that of the $\mathrm{ChLC}$ helix for the both laser modes observed at the two PhBG edges $\lambda_{\|}$and $\lambda_{\perp}$.

The mirrorless lasing in $\mathrm{Sm} C^{*}$ has been reported in Ref. [29]. The first lasing in a 3D PhC comprised of the LC phase called as BPII has been demonstrated in Ref. [30]. Later on, the same has been reported for the phase of the type BPI [31,32]. The pumping intensity threshold is almost twice as lower than that for the ChLC samples with the same dye concentration and under the same pumping conditions. Highly directional distributed-feedback lasing has been observed in the three mutually perpendicular directions.

There are other LC phases which might be examined for lasing. These are twist grain-boundary phases [5], smectic Q [33] and defect patterns in smectics with the photonic structure, in particular a periodical array of focal conic domains in the hybrid aligned smectic A films [34, 35]. Lyotropic chromonic LCs (LCLC), most of which are dyes, also represent promising materials for the optically pumped mirrorless lasing [36, 37]. There are two types of chromonic aggregates, $\mathrm{H}-$ and $\mathrm{J}$-aggregates. Molecules in the $\mathrm{H}$-aggregates are stacked such that their molecular planes are perpendicular to the long axis of the aggregate. Each next molecule in the J-aggregates is shifted in the same lateral direction with respect to the other molecules in the aggregate, such that the molecular planes appear to be tilted by some angle with respect to the long axis of the aggregate. It appears that only the J-aggregates show photoluminescence. Moreover, it turns out that the J-aggregates show the narrowest absorption and luminescence bands among the other organic dyes, a large value of the oscillator strength, a giant increase of the emission rate in the aggregated state with respect to the emission in a monomer state and, probably, the highest third-order nonlinear susceptibility $[38,39]$. Coherent coupling between localized plasmons of a nanoshell and excitons of the molecular J-aggregates adsorbed on the surface has been implemented in Ref. [40]. To our knowledge, still there are no reports on the lasing occurring in the LC phase of lyotropic chromonics.

The lasing effect in the dye-doped cholesterics has been patented by Goldberg and Shnur [41] as long ago as in 1973. The first theoretical analysis of the ChLC laser is due to Kukhtarev [42], who has predicted in 1978 a low lasing threshold for this laser in comparison with the other distributed-feedback lasers. Lasing in the dye-doped ChLC has been first evidenced in 1980 by Iltchishin et al. [43]. Fifteen years later Iltchishin and Vakhnin [44] have documented lasing in the dye-doped ChLC, with the two wavelengths located at the edges of the SLR. However, at the time the importance of this finding, which unambiguously points to the PhBG edge mechanism of the lasing in ChLCs, has not yet been recognized. Instead it has been explained as a consequence of a distorted structure of the cholesteric helix. The first clear proof of the PhBG edge mechanism of the lasing in ChLCs has been presented in 1998 by Kopp et al. [45]. They have demonstrated unequivocally that the lasing line coincides with the short-wavelength edge of the PhBG and that the emitted laser light is circularly polarized, with the same chirality sign as that of the cholesteric helix. 
Soon Taheri et al. [46, 47] have reported lasing at the long-wavelength side of the PhBG for a different ChLC-dye system. It is interesting that in the works [46, 47] the lasing has also been observed with no doping of ChLC by dye molecules, and a pure ChLC has been pumped with the UV light. In this case the ChLC plays a dual role: it is a gain medium and provides simultaneously a distributed feedback.

It seems now surprising enough that, although it has been known well before the discovery of the ChLC lasers that the cholesteric PhBG reveals a blue-shift with increasing viewing angle $\beta$ ( $\lambda_{m}=\bar{n} P \cos \beta$, with $\lambda_{m}$ being the spectral middle of the PhBG and $\bar{n}=\sqrt{n_{\|}^{2}+n_{\perp}^{2}}$ ), it has been widely accepted for a long time that the lasing in the dye-doped ChLCs can be observed only along the helical axis (see, e.g., Ref. [48] and the more recent review [49]). Lack of evidence that the feedback necessary for the true lasing [2] can be achieved at an oblique (off-axis) light emission has been, probably, one of the main sources for this belief. Only in 2006, Palto [50] has unambiguously demonstrated that the Bragg lasing conditions can be satisfied not only for the lasing along the helical axis, but also for a wide range of oblique angles. He has argued that, for the oblique light-emission angles, the existence of the feedback loop necessary for the laser generation is assured by a smallness of thickness of the ChLC cell in comparison with its lateral dimensions. Further on, we will call the Bragg lasing regimes along or off the helical axis respectively as in- or off-axis Bragg (PhBG) lasing. The ring shape of a laser spot often observed [51, 52] on the screen behind the cholesteric lasers, which was believed to be a diffraction pattern [51] or explained as interference fringes [48], has been identified in Ref. [50] as a natural feature of the off-axis Bragg lasing. A further peculiarity distinguishing the off-axis Bragg lasing from the multiple reflection interference is that, in case of the latter effect, the pattern on a screen is formed by the light of the same wavelength, whereas the wavelength is expected to decrease towards the periphery of the pattern in case of the off-axis Bragg lasing. Experimentally the off-axis Bragg lasing in a dye-doped ChLC observed as concentric rings of decreasing wavelength on the periphery has been proven for the first time in Ref. [53].

Later on, the off-axis Bragg lasing in the dye-doped ChLCs has been reported in Refs. [54-57] and termed as a colour-cone lasing. Detailed theoretical and experimental investigations of the off-axis Bragg lasing has recently been performed by Palto et al. [58] and Pennick et al. [59]. One of the important conclusions following from Ref. [58] is that the off-axis lasing mode, together with a waveguide mode $W_{g}$ and so-called quasi-in-plane leaky (QIPL) modes, are the most important mechanisms competing with the conventional in-axis Bragg laser mode for the emission power. The $W_{g}$-mode is based on the effect of total internal light reflection and thus takes place only if the refractive index of substrates is lower than at least one of the refractive indices $n_{\perp}$ or $n_{\|}$of a LC for the angles of light propagation larger than a corresponding threshold angle of the total internal reflection. The QIPL modes are due to a strong Fresnel reflection at substrates occurring for the propagation angles close to $90^{\circ}$ with respect to the normal of the substrates. The QIPL modes have been initially identified in Ref. [60] and studied in details in Ref. [61]. The off-axis Bragg mode, the $W_{g}$-mode and the QIPL modes affect significantly the in-axis Bragg lasing mode, thus increasing its threshold and lowering its efficiency. It is argued in Ref. [62] that the 'parasitic' effect of these modes can be mitigated when the cholesteric helix axis is aligned in the cell plane.

All of the ChLC lasers known up to date are pumped with pulse lasers. There is still no report in the literature on a continuous-wave lasing in the dye-doped ChLCs. The main problem is that, although the pumping threshold in the ChLC lasers is significantly lower than that in the other mirrorless media, it is still too high to avoid a damage of cholesteric structure occurring under 
continuous-wave pumping. Morris et al. [63] have shown that the lasing intensity drops by about ten times when the repetition rate increases from $1 \mathrm{~Hz}$ to $20 \mathrm{~Hz}$ at a constant pumping intensity. Their results indicate that the typical pumping energies at which the ChLC lasers are operated are sufficient for accumulating light-induced director reorientation. Thus, decreasing of the threshold pumping energy, desirably to zero, is highly demanded and all the research efforts on the ChLC lasing seem to be stimulated by this goal in one or another way.

Since the ChLC laser is a laser with distributed feedback, its threshold can be estimated using the expression derived by Kogelnik and Shank [10] $\left(E_{t h}=A\left[\chi d+\lambda^{2} /\left(\Delta n^{2} d^{2}\right)\right]\right.$, where $\chi$ is the absorption coefficient). It is seen from this expression that $E_{t h}(d)$ is a nonmonotonic function, which has a minimum. The experiments $[64,65]$ have confirmed this prediction, giving an optimal value for the thickness of the ChLC cell ( $d \sim 15 \mu \mathrm{m})$. Another important conclusion following from the theoretical $E_{t h}(d)$ dependence is that increasing $\Delta n$ implies lowering $E_{t h}$. This is a direct consequence of the dependence of $E_{t h}$ on the maximal photon density of states $\rho$ at the PhBG edge derived in Refs. $[64,65]\left(E_{t h}=A \chi d+(B / \rho)\right)$. Indeed, on the one hand, the density of states at the band edge is an increasing function of the birefringence [66] and, on the other hand, we have $\rho=C d^{2}$ [64] (with $A, B$ and $C$ being constants). Since $\Delta n$ is proportional to the scalar orientational order parameter $S$ for the LC molecules, $E_{t h}$ is expected to decrease with increasing $S$.

Different approaches have been developed to reduce the lasing threshold, in particular operation in different lasing modes. These are the off-axis Bragg mode, the $W_{g}$ and QIPL modes, and the in-plane alignment of the cholesteric axis [62] mentioned above. The other possibilities are from the defect modes considered in Sec. III C, a synthesis of new highly effective dye materials [67, 68], a use of so-called Förster energy transfer [69-73] from a donor to acceptor-dye molecules, enhancement of the scalar orientational order parameter of dye [68] and LC [74-76] materials, and an increase in the birefringence of LC matrices [77].

Since the ChLCs are anisotropic media, their lasing efficiency should depend on polarization state and incident angle of the pumping beam. These dependences are governed by the processes of photoexcitation and light losses. The results presented in Ref. [78] suggest that the pumping is more efficient for smaller incident angles because of lower reflection. For higher incidence angles it is more efficient to pump with the circularly polarized light of the handedness opposite to that of the ChLC. Thus one can avoid the selective reflection of the pumping light when its wavelength overlaps with the PhBG, since the PhBG moves toward shorter wavelengths with increasing incident angles [78].

The main parameters characterizing the performance of the ChLC lasers are the excitation (pumping) threshold energy $E_{t h}$ and the slope efficiency. Notice that usually the latter is inversely proportional to $E_{t h}$ [32]. Multiple stacking of dye-doped chiral polymer films [79] and amplification of laser emission from the dye-doped ChLCs by a uniform dye-doped nematic LC layer [80] have been suggested to increase the lasing power.

Wang et al. [81] have studied the relationship between the threshold power and the excitation wavelength for the dye-doped ChLC lasers and found that the threshold power at the short-wavelength tail of the absorption band is almost an order of magnitude lower than at the long-wavelength tail, thus indicating to existence of an optimum wavelength that gives rise to the lowest threshold. The performances of the ChLC lasers with different dyes have recently been compared in Ref. [82]. Nevertheless, the problem of lowering of the pumping threshold is still far from its solution. 


\subsection{Tuning of low-molecular cholesteric liquid-crystal lasers}

Significant research effort in the area of ChLC lasers has been concentrated on spectral tuning of the lasing lines. Several different approaches have been used for this aim. The main parameter controlling the spectral position of the lasing line is the cholesteric pitch $P$, which defines the position of the cholesteric PhBG. The cholesteric pitch can be tuned by temperature, concentration of a chiral dopant, or external electric field. In ChLC elastomers, $P$ can be varied by mechanical strains. If the twisting power of a chiral dopant is sensitive to the optical (e.g., UV) irradiation, the pitch and therefore the lasing can be phototunable [52, 83, 84]. It is worth noticing that a chiral dopant ZLI-811 (also known as S811) undergoes a photo-transformation under UV irradiation with the wavelengths shorter than $300 \mathrm{~nm}$ [83], which leads to decreasing twisting power and thus moving the PhBG towards the longer wavelengths. Spatial tuning of the lasing may be implemented if a gradient of the pitch is formed in a dye-doped ChLC sample, moving the spot of the pumping light along the pitch gradient direction. The pitch gradient can be produced via placing in contact two ChLC mixtures with different concentrations of the chiral agent [83], by photo-patterning [85], or through a temperature dependence of solubility of the chiral agent [86]. A position-sensitive dye-doped ChLC laser covering a whole visible range has been fabricated by the authors of Ref. [87].

Temperature tuning can be direct (due to a temperature dependence of the pitch as a consequence of temperature-dependent elastic LC properties) or indirect (e.g., through a temperature-dependent solubility of the chiral agent [86]). The approach of oversaturated chiral agents provides multi-wavelength lasing [88]. Non-uniform solubility leads to a multidomain structure, in which the cholesteric pitch becomes different in different domains. The size of the domains can be controlled by the cooling rate. In this way, up to nine laser wavelengths have been simultaneously observed in Ref. [88]. It is interesting that the temperature variation of the pitch is not continuous, due to strong anchoring at surfaces in thin confined samples, and the pitch changes by the steps $P / 2$. As a result, the temperature tuning of the laser line in such ChLC samples is discontinuous, whenever the size of the pumping spot is small enough [89]. As shown in Ref. [90], the $\lambda(T)$ discontinuity can be removed while combining two chiral doping agents that exhibit the opposite temperature dependences for the pitch. Temperature variation of the order parameter can also be used for enhancing the efficiency of the ChLC lasers [74].

Unfortunately, smooth electric-field-induced unwinding of the cholesteric pitch predicted by de Gennes [91] is preceded by the other field-induced structural instability effects. All that can be done with application of the electric field is gradual reduction of the emission intensity down to a total lasing quenching. Some promising results evidencing that the lasing wavelength of the dye-doped ChLC laser can be tuned in the range of about $10 \mathrm{~nm}$ with an in-plane electric field have been reported recently [32]. Finally, an electrically tunable waveguide laser based on ferroelectric LCs has been demonstrated in Ref. [92].

Due to the Stocks shift in the conventional dye-doped ChLC lasers, the lasing wavelength is longer than the pumping wavelength. The situation is the opposite for the two-photon lasing. For instance, the pumping at the wavelength of $1000 \mathrm{~nm}$ leads to the lasing at the cholesteric band edge (i.e., at $608 \mathrm{~nm}$ ) [93]. The laser emission from a dye-doped ChLC pumped by another ChLC laser has been demonstrated in Ref. [94].

\subsection{Polymer cholesteric liquid-crystal lasers}

Lasing has been observed not only in the low-molecular cholesterics but also in polymer ChLCs (PChLCs). A PChLC represents a promising laser material since it is expected to reveals the properties which are not available in the low-molecular ChLCs. One of the advantages of the PChLCs is their stability under high-power pumping, contrary to the low-molecular ChLCs that 
suffer from significant distortions of their structure at high pumping powers, thus worsening and finally destroying the laser generation. To overcome this problem, a stiff PChLC has been synthesized and doped with a dye [95]. To enhance the resistance to high-power optical irradiation, the polymer chains have been cross-linked into a network [96]. The lasing characteristics of such a PChLC have been compared to those of a low-molecular ChLC doped with the same dye. As expected, the cross-linking effectively protects the LC material against a light-induced destruction of the cholesteric order.

Surprisingly, the other lasing characteristics have also appeared to be much better (e.g., a lower pumping threshold, almost an order of magnitude higher maximum laser intensity observed at the same pumping, and almost twenty times higher slope efficiency). These facts indicate that the cross-linking improves the local cholesteric order. Interestingly enough, the lasing spectrum of the PChLC contains several lines, the availability of which has been assigned to imperfect macroscopic cholesteric orientation over the sample. The number of the laser lines can be different, depending on the position of pumping spot. In elastomer PChLCs, the pitch of the cholesteric helix and, consequently, the spectral position of the lasing line can be tuned by applying a strain [97]. Another advantage provided by the PChLCs is that the polymer films are flexible and can be bent [98]. A polymer dye aligned parallel to the $\mathrm{ChLC}$ molecules has been used to enhance the lasing efficiency [99].

\subsection{Defect modes in cholesteric liquid-crystal lasers}

It is remarkable that the introduction of defects in the PhCs results in extra modes. They appear inside the PhBG where the normal modes of a perfect $\mathrm{PhC}$ are inhibited [14]. The defect modes are a direct consequence of localization of photons on the defects, and a single defect in a $\mathrm{PhC}$ is akin to a Fabry-Pêrot cavity, except that it is omnidirectional and multiply reflects radiation in the overall spatial angle $4 \pi$ [100]. In a PhC structure made of a solid dielectric material, a defect can be created while adding/removing some material to/from a unit cell. Such a defect behaves similarly to a donor/acceptor atom in a semiconductor and leads to donor/acceptor modes below the conduction/above the valence band. It has been demonstrated [100] that the donor/acceptor modes appear inside the PhBG near its short-/long-wavelength edge. Not less remarkable is that the lasing threshold for a defect mode appears to be lower than that for the band-edge modes. Random defects in a $\mathrm{PhC}$ give rise to localized states due to the multiple scattering [12, 101, 102].

For ChLCs it has been suggested to create defects by replacing a thin ChLC layer by an isotropic material [103] or by a nematic layer $[104,105]$. When introducing a dye-free nematic layer sandwiched between two dye-doped polymer ChLC films with the same handedness of the cholesteric helix but different pitches [105], one can tune the phase retardation of the nematic layer with applying voltage and, thereby, control the intensity of the laser light generated in the forward and backward directions [106]. Such a device can work in a regime of optical diode, manifesting unidirectional emission for one of the circular components. In Ref. [105], a nematic cell doped with a dye has been sandwiched between two dye-free cholesteric cells and the phase retardation of the nematic cell has been controlled with the voltage, thus allowing for both the intensity and the spectral position controls of lasing. It has also been suggested that a defect can be created by twisting one part of a chiral photonic structure about its helical axis [107], thereby introducing a phase jump in the cholesteric helix. This idea has been successfully verified in the experiments [108] for the two PChLC films mutually rotated by $90^{\circ}$ about their helical axes normal to their faces, and then placed in close contact.

A combination of both the 'isotropic' and 'twist defect' approaches has been considered theoretically by Schmidtke and Stille [109], who have presented an analytical approach in the frame of theory of defect modes in helical PhCs [110]. The authors have argued that even very small 
absorption destroys the optical effects induced by defects in the photonic cholesteric structures, leading to unexpected effects, e.g., a decrease in the total absorption inside thick samples occurring when the absorption coefficient increases above some value. Basing on a numerical analysis, it has been suggested [111] that the introduction and tuning of the photonic defect modes in ChLCs can be realized via optically induced local deformation of the cholesteric structure, such that a local shortening (elongation) of the helix leads to a blue (red) shift of the defect lasing mode. The defect-mode lasing with a three times reduced threshold has been reported for the structures composed of three cells $[112,113]$. Multilayered structures of the dye-doped ChLCs used in the study [114] have revealed lasing thresholds as low as $12 \mathrm{~nJ} /$ pulse, which is an order of magnitude lower than that for the equivalent single-layer $\mathrm{ChLC}$ cell. Introduction of defects of certain sequences such as Fibonacci [115-117], Thue-Morse [118], Rudin-Shapiro [119], etc. is attractive for fabricating the photonic structures with the multiple PhBGs. In particular, they can be used as cavities for the mirrorless lasers.

It is believed that poor LC alignment worsens the performance of ChLC lasers. On the other hand, as discussed above in this subsection, incorporation of defects into the cholesteric structure can bring about some new features and lower the pumping threshold. The question of how the presence of a cholesteric disclination influences the lasing remains open and rather intriguing. Coles et al. [32] have demonstrated that a poorly aligned dye-doped cholesteric cell shows multiple overlapping lasing modes, which apparently look as broadening of the laser line from about $0.1 \mathrm{~nm}$ (for the case of good alignment) to several nanometres (for poorly aligned samples). Now it is well established that the LC defects are useful for generation of optical singularities [120, 121]. Then the lasing in the dye-doped ChLCs with defects can be of interest while generating singular beams.

To finish this section, we stress that the laser generation in dye-doped ChLCs is based on light amplification in a naturally existing resonator based on the selective multiple Bragg reflection of light from a periodic cholesteric structure. As mentioned in the Introduction, there is another possibility to obtain the resonance, using the total internal reflection effect in the closed cavities. This mechanism of mirrorless lasing is a subject of the next section.

\section{Lasing in microcavities}

Optical microcavities exist in diverse geometrical shapes and embrace the objects of dimensionalities ranging from $D=1$ (fibres and optically stratified media) up to $D=3$ ('closed figures' such as a sphere, disc or a toroid). One of the key requirements for such resonators is that their refractive index must be higher than that of a surrounding medium. Another restriction is set by the resonance conditions upon their size. In addition, to get high $Q$ values, their surfaces must be as smooth as possible, desirably on the scales as small as nanometres. All of these requirements are met in artificially produced closed microcavities, for which the smoothness of the surface is provided during their fabrication by a surface tension or by fine polishing. For example, silica microspheres show the $Q$-factors around $10^{10}$, the $Q$-factor for the toroidal silica microcavities with the dimensions of the order of $100 \mu \mathrm{m}$ is well above $10^{8}$, the highest value for the silica microdiscs is about $6 \times 10^{7}$, whereas the $Q$-factor for the liquid droplets is less than $10^{5}$ [122].

Since the resonances in the closed microcavities are strongly governed by the resonator shape, their modes are called as morphology-dependent resonant modes. Historically they are also termed as whispering-gallery modes (WGM). This name is referred to the effect of sound-wave propagation observed in the Whispering Gallery of St. Paul's Cathedra, where even a whisper is audible to a listener at any point along the gallery. The St. Paul's Cathedra was officially opened in 1711 and, two centuries later (in 1910-1912), the whispering-gallery effect has been identified by Lord Rayleigh (John William Strutt) [123] as resonant refocusing of the sound waves by curved surfaces. 
It has been recognized later that the effect is not inherent to the sound alone but applies to the waves of any nature, including the light ones.

Mirrorless optical microcavities [124] working on the WGM principle have been fabricated as solid microparticles of different shapes (spheroids, toroids and discs), or liquid droplets. It is worth noticing that, according to Ref. [122], cylindrical and, in some cases, ring microresonators that act as optical fibres, are not whispering-gallery resonators in the strict sense, if their curvature does not play a key function in forming the resonances. The simplest 3D WGM optical resonators are dielectric microspheres [125]. Due to low optical losses and small mode volumes, the microresonators provide very high quality factors (up to $10^{9}[126]$ ) and the lasing thresholds as low as $200 \mathrm{nW}$ [127]. The microspheres are easily produced in a laboratory. One of the possibilities is to melt a fibre end such that a single sphere appears to be attached to the fibre, which is convenient for both study and applications. The idea of using the spherical resonators for lasing is due to Garrett et al. [128]. To produce laser generation, the microspheres fabricated from isotropic (glass [129], silica $[127,130]$, polymers [131], etc.) or crystalline materials [128] can be either doped with luminescent elements (such as rare-earth ions), or coated with a fluorescence dye, or supplied with embedded or attached quantum dots. The laser generation has been demonstrated in Ref. [132] for hollow semiconductor microspheres with bilayer walls containing closely packed $\mathrm{Cd} / \mathrm{Se}$ quantum dots in their inner layer and silica nanoparticles in the outer layer. The WGM resonances have also been observed in the fluorescence of microspheres coated with nanolayers of a lyotropic chromonic LC pseudoisocyanin, which form J-aggregates on their surfaces [39, 133].

In the same vein, the WGM resonances and, consequently, the low-threshold lasing can be obtained in the microdiscs fabricated of or doped with a gain material. Similarly to the case of microspheres, where the WGM lasing is omnidirectional in the equatorial plane, the direction of the WGM lasing in circular microdiscs is also degenerated, being confined in the plane parallel to the disc plane. This feature may be considered as advantage for some applications, though in some other cases it may cause serious drawbacks. Considerable efforts have been concentrated on breaking this symmetry feature $[134,135]$ by deforming the shape of microspheres [136] and microdiscs [137, 138]. Different deformations have been suggested for the microdiscs, of which only some lead to unidirectional emission [138]. Finally, the microlasers can also be based on liquid droplets acting as spherical mirrorless resonators. This subject will be reviewed in the next subsection.

\subsection{Lasing droplets}

Lasing droplets can be freely suspended in another immiscible isotropic liquid, embedded in a polymer matrix, or flowing with a matrix liquid along microchannels. Static liquid suspensions are convenient for experimental studies, because of ideally spherical shape of their freely suspended droplets. The shape of the droplets in polymer dispersions is usually distorted and so their theoretical description is complicated. In addition, the quality factor of such imperfect droplet resonators significantly lowers, thus increasing the lasing threshold. The polymer droplet dispersions can serve as paintable laser-lightening films.

A considerable attention has recently been paid to the fabrication of optofluidic systems and, in particular, of microfluidic dye lasers [139]. These lab-on-a-chip devices carry out the main functions of their conventional bulky counterparts. As a rule, they represent miniature versions of the latter and, at the same time, provide novel possibilities for applications. According to the on-chip design, the constructive elements of the laser (including mirror-based resonators, mixers, microchannels, etc.) for the flowing dye solution are fabricated with a soft lithography [140-143]. An interesting design of a collinear single-mode dual-colour dye microlaser has recently been suggested in Ref. [144], where an on-chip Fabry-Pêrot cavity is combined with a system producing a periodic 
stream of droplets containing alternatively the dyes Rhodamin B and Rhodamin 6G. This very example concerns the lasing with a traditional mirror-based resonator though, in principle, both mirror-based and mirrorless feedbacks can be implemented in the microfluidic dye lasers. The on-chip microfluidic lasers can be integrated in microdevices. When compared with their static counterparts, the advantage of the microfluidic dye lasers is that the flowing droplets suffer considerably less from the parasitic light-induced effects such as photo-bleaching, light-induced overheating and structural damage.

Additional interesting features appear if the lasing droplets are in the LC state. The WGM lasing in the dye-doped nematic droplets has been demonstrated in Ref. [145], with the surface director orientation being continuously varied from planar to homeotropic. High sensitivities of LCs to the external fields allow for controlling their optical properties with the electric fields applied. The anisotropic light emission in the dye-doped PDLC controlled by the electric fields has been reported in Ref. [146]. Though a 'random laser' mechanism (see the first part of our review [2]) of anisotropic light emission observed in the dye-doped nematic droplets embedded in a polymer matrix is quite plausible and intriguing (see [146]), it would be desirable to examine it against the other lasing modes which can exist in such a complicated system. The presence of different lasing modes has been analyzed by Liu et al. [147] in a similar experiment, though with the PDLC droplets of nanometre scales placed in a significantly thinner $(6 \mu \mathrm{m})$ cell. The light emission collected from the edge of the cell has been observed in Ref. [147], with the spectrum containing a relatively broad band. The emission band narrows from $\sim 40 \mathrm{~nm}$ to $13 \mathrm{~nm}$ and displays sharp narrow $(<1 \mathrm{~nm}$ in their width) spikes when the pumping intensity increases from 25 to $55 \mu \mathrm{J} /$ pulse. It is these sharp spikes observed against the background of a broad amplified spontaneous emission band that have been qualified as being due to the 'random lasing'. It has been argued that the emission collected from the cell edge might be greatly influenced by the waveguide mode, though one has to keep in mind that the quasi-in-plane leaky modes and the WGM modes might have significant contributions, too.

Since the ChLC represents a PhBG resonator per se, it is highly intriguing to study the lasing in the dye-doped ChLC droplets dispersed in an immiscible isotropic liquid. The idea has been realized by Humar and Muševič [148]. To suppress the WGM mode, the LC has been chosen to match its refractive index with that of the glycerol used as a matrix for the LC droplets, and the birefringence of the LC material has been small $(\Delta n=0.07)$. It is important that the glycerol provides a parallel anchoring for the LC molecules at the droplet interface, such that the cholesteric layers are roughly spherically concentric. Due to topological constraints, the structure contains either one disclination line of the topological strength $s=2$ or two disclinations, each of the strength $s=1$ ) running from the droplet surface to its centre, where they are attached to a central point defect.

Topologically such a structure is akin to the Dirac magnetic monopole [5, 149]. The radially oriented cholesteric helical axis is an analogue of the magnetic field strength and the LC director corresponds to the vector-potential of the magnetic field. A pumping threshold has been clearly detected. It has been found to be extremely low (about $20 \mathrm{~nJ}$ ) for the case when the $1 \mathrm{~ns}$ long pumping pulse is focused to a spot uniformly illuminating the droplet ( $40 \mu \mathrm{m}$ in the diameter). The pumping threshold increases with decreasing droplet diameter. The increasing rate is slow for the diameters larger than $50 \mu \mathrm{m}$ and rapid for the smaller droplets. The smallest lasing droplet has been found to be $15 \mu \mathrm{m}$ in diameter. The light emission above the pumping threshold is typical of the cholesteric PhBG edge lasing, except for that the emitted light is essentially depolarized (the polarization is neither circular nor linear). The laser line detected at the long-wavelength edge of the $\mathrm{PhBG}$ is defined by the cholesteric pitch and is temperature tunable for about $30 \mathrm{~nm}$. The lasing is omnidirectional, whereas the emitted intensity and the wavelength measured for one droplet are independent of the viewing angle. The width of the laser line is extremely narrow (less than 
$0.05 \mathrm{~nm}$ ). Though the lasing has not been studied along the disclination line, one can expect that the presence of the defect might bring about new modes, in line with the discussion in Sec. III C.

It has been found $[150,151]$ that in the materials exhibiting a so-called TGBA phase between the cholesteric and smectic A phases, the central point defect splits into a loop at the transition cholesteric-TGBA. It would be interesting to study the influence of such a structural transformation on the lasing in droplets with initially spherically symmetric cholesteric layers. Notice that a photosensitive chiral azo-dopant has been used in Ref. [152] to tune the wavelength and switch the lasing intensity in the droplet dye-doped ChLC laser.

\section{Final comments}

Let us summarize the main conclusions of the present article and make the final comments on the subject. This article represents the second part of the review on optically pumped mirrorless lasing, where consideration of the random lasing presented in the first part [2] is complemented by analysis of the literature on the lasing in photonic structures and microcavities. Besides of these three branches elaborated during the last two decades, there is also a recent discovery of the mirrorless lasing in SPASERs, surface nanoplasmonic counterparts of the mirrorless lasers, which show rapid and very promising developments.

It is impressive that the development of lasers reveals the tendencies which are common with many other devices - from bulky monsters to miniature contraptions. It is very similar to the progress seen for the computers: old computing machines occupying several rooms have eventually transformed into desktop personal computers, portable laptops and a variety of smart pocket-size devices, owing to a tremendous progress occurred in semiconductor physics and technology. Quite similarly, lasers as bulky devices have been miniaturized and released from an optical bench, where they were born and exploited for a long time, by invention of a luminescent semiconductor as a gain medium. Exclusion of the mirrors from the construction of lasers is a next step in their progress. This simplifies the production, reduces the cost, and makes the lasers portable.

In its turn, optical pumping makes the mirrorless lasing process wireless, thus greatly extending the possible applications. Each of the optically pumped mirrorless mechanisms known to date implies its specific possibilities for the further improvement, although lowering of the pumping threshold to a desirable level of zero is a common goal of all of them. To our knowledge, the dye-doped LC lasers manifest the lowest pumping thresholds among various optically pumped mirrorless lasers. Besides of the optical losses caused by different imperfections, which represent the main worsening factor common to all lasers, the competition of different lasing modes for the emitted light power seems to be of the most considerable importance for the LC lasers. The latter may be illustrated by the lasing of dye-doped ChLC droplets dispersed in immiscible liquids, which reveals the thresholds as low as $20 \mathrm{~nJ} /$ pulse, due to a perfectly smooth interface and a single-mode PhBG lasing.

\section{References}

1. Purcell E M, 1946. Spontaneous emission probability at radio frequencies. Phys. Rev., Proc. Amer. Phys. Soc. Abstract B10. 69: 674-702.

2. Nastishin Yu A and Dudok T H, 2013. Optically pumped mirrorless lasing. A review. Part I. Random lasing. Ukr. J. Phys. Opt. 14: 146-170.

3. Stokes G G, 1885. On a remarkable phenomenon of crystalline reflection. Proc. Roy. Soc. 26: 174-186.

4. Rayleigh J W S, 1888. On the remarkable phenomenon of crystalline reflexion described by Prof. Stokes. Phil. Mag. 26: 256265.

Ukr. J. Phys. Opt. 2014, Volume 15, Issue 2 
5. Kleman M, Lavrentovich O D and Nastishin Yu A, Dislocation and disclination in mesomorphic phases, Vol. 12, in 'Dislocations in Solids', Ed. by F R N Nabarro and J P Hirth, Elsevier, 147-271 (2004).

6. Kleman M, Meyer C and Nastishin Yu A, 2006. Imperfections in focal conic domains: the role of dislocations. Phil. Mag. 86: 4439-4458.

7. De Gennes P G and Prost J, The physics of liquid crystals, $2^{\text {nd }}$ Ed. Oxford: Clarendon Press (1993).

8. Kogelnik H and Shank C V, 1971. Stimulated emission in a periodic structure. Appl. Phys. Lett. 18: $152-154$.

9. Shank C V, Bjorkholm J E and Kogelnik H, 1971. Tunable distributed feedback dye laser. Appl. Phys. Lett. 18: 395-396.

10. Kogelnik H and Shank C V, 1972. Coupled wave theory of distributed feedback lasers. J. Appl. Phys. 43: 2327-2335.

11. Yablonovitch E, 1987. Inhibited spontaneous emission in solid-state physics and electronics. Phys, Rev. Lett. 58: 2059-2062.

12. John S, 1984. Electromagnetic absorption in a disordered medium near a photon mobility edge. Phys. Rev. Lett. 53: 2169-2172.

13. John S, 1987. Strong localization of photons in certain disordered dielectric superlattices. Phys. Rev. Lett. 58: 2486-2489.

14. John S, 1991. Localization of light. Phys. Today. 44: 32-40.

15. Yablonovitch E, 2001. Photonic crystals: Semiconductors of light. Scientific American. 285: 47-55.

16. Dowling J P, Scalora M, Bloemer M J and Bowden Ch M. 1994. The photonic band edge laser: A new approach to gain enhancement. J. Appl. Phys. 75: 1896-1899.

17. John S and Quang T, 1991. Localization of superradiance near a photonic band gap. Phys. Rev. Lett. 74: 3419-3422.

18. Baba T, 2008. Slow light in photonic crystals. Nature Photonics. 2: 465-473.

19. Megens M, Wijnhoven J E G J, Lagendijk Ad and Vos W L, 1999. Light sources inside photonic crystals. J. Opt. Soc. Amer. B. 16: 1403-1408.

20. Bulu I, Caglayan H and Ozbay E, 2003. Radiation properties of sources inside photonic crystals. Phys. Rev. B. 67: 205103-7.

21. Wu S-T and Fuh A Y-G, 2005. Lasing in photonic crystals based on dye-doped holographic polymer-dispersed liquid crystal reflection gratings. Jpn. J. Appl. Phys. 44: 977-980.

22. Woltman S J and Crawford G P, 2007. Patterned liquid-crystal laser film for multi-dimensional multi-color emissive film technology. J. SID. 15/8: 559-564.

23. Jakubiak R, Natarajan L V, Tondiglia V, He G S, Prasad P N, Bunning T J and Vaia R A, 2004. Electrically switchable lasing from pyrromethene 597 embedded holographic-polymer dispersed liquid crystals. Appl. Phys. Lett. 85: 6095-6097.

24. Strangi G, Barna V, Caputo R, De Luca A, Versace C, Scaramuzza N, Umeton C, Bartolino R and Price G N, 2005. Color-tunable organic microcavity laser array using distributed feedback. Phys. Rev. Lett. 94: 063903-4.

25. Liu N, Guo H, Fu L, Kaiser S, Schweizer H and Giessen H, 2008. Three-dimensional photonic metamaterials at optical frequencies. Nature. 7: 31-37.

26. Kobayashi Ch, Yamamoto J and Takanishi Y, 2012. Photonic effect in a hyper-swollen lyotropic lamellar phase. J. Appl. Phys. 112: 013531.

27. de Vries Hl, 1951. Rotatory power and other optical properties of certain liquid crystals. Acta Cryst. 4: 219-226. 
28. Schmidtke J and Stille W, 2003. Fluorescence of a dye-doped cholesteric liquid crystal film in the region of the stop band: theory and experiment. Eur. Phys. J. B. 31: 179-194.

29. Ozaki M, Kasano M, Ganzke D, Haase W and Yoshino K, 2002. Mirrorless lasing in dye-doped ferroelectric liquid crystal. Adv. Mater. 14: 306-309.

30. Cao W, Munoz A, Palffy-Muhoray P and Taheri B, 2002. Lasing in a three-dimensional photonic crystal of the liquid crystal blue phase II. Nature. 1: 111-113.

31. Yokoyama S, Mashiko S, Kikuchi H, Uchida K and Nagamura T, 2006. Laser emission from a polymer-stabilized liquid crystal phase. Adv. Mater. 18: 48-51.

32. Coles H J, Morris S M, Ford A D, Hands P J W and Wilkinson T D, 2009. Red-green-blue 2D tuneable liquid crystal laser devices. Proc. SPIE. 7414: 741402-21.

33. Pansu B, Nastishin Y, Imperor-Clerc M, Veber M and Nguyen H T, 2004. New Investigations on the tetragonal liquid crystalline phase or SmQ. Eur. Phys. J. E. 15: 225-230.

34. Ruan L Z, Sambles J R and Stewart I W, 2003. Self-organized periodic photonic structure in a nonchiral liquid crystal. Phys. Rev. Lett. 91: 033901-4.

35. Pishnyak O P, Nastishin Yu A and Lavrentovich O D, 2004. Comment on 'Self-organized periodic photonic structure in a nonchiral liquid crystal'. Phys. Rev. Lett. 93: 109401-1.

36. Lydon J, 2011. Chromonic liquid crystalline phases. Liq. Cryst. 38: 16631681.

37. Nastishin Yu A, Liu H, Schneider T, Nazarenko V, Vasyuta R, Shiyanovskii S V and Lavrentovich O D, 2005. Optical characterization of the nematic lyotropic chromonic liquid crystals: light absorption, birefringence, and scalar order parameter. Phys. Rev. E. 72: 041711-14.

38. Kobayashi T, Ed., J-aggregates. Singapore: World Scientific (1996).

39. Melnikau D, Savateeva D, Chuvilin A, Hillenbrand R and Rakovich Yu P, 2011. Whispering gallery mode resonators with J-aggregates. Opt. Express. 19: 22280-22291.

40. Fofang N T, Park T-H, Neumann O, Mirin N A, Nordlander P and Halas N J, 2008. Plexcitonic nanoparticles: plasmon-exciton coupling in nanoshell-J-aggregate complexes. Nano Lett. 8: 3481-3487.

41. Goldberg L S and Shnur J M, 1973. Tunable internal-feedback liquid. U.S. Pat. No 3,771,065.

42. Kukhtarev N V, 1978. Cholesteric liquid crystal laser with distributed feedback. Sov. J. Quantum Electron. 8: 774-776.

43. Il'chishin I P, Tikhonov E A, Tishchenko V G and Shpak T M, 1981. Generation of tunable radiation by impurity cholesteric liquid crystals. JETP Lett. 32: 24-27.

44. Ilchishin I P and Vakhnin A Yu, 1995. Detecting of the structure distortion of cholesteric liquid crystal using the generation characteristics of the distributed feedback laser based on it. Mol. Cryst. Liq. Cryst. 265: 687-697.

45. Kopp V I, Fan B, Vithana H K M and Genack A Z. 1998. Low-threshold lasing at the edge of a photonic stop band in cholesteric liquid crystals. Opt. Lett. 23: 1707-1709.

46. Taheri B, Munoz A F, Palffy-Muhoray P and Twieg R, 2001. Low threshold lasing in cholesteric liquid crystals. Mol. Cryst. Liq. Cryst. 358: 73-82.

47. Munoz A F, Palffy-Muhoray P and Taheri B, 2001. Ultraviolet lasing in cholesteric liquid crystals. Opt. Lett. 26: 804-804.

48. Ford A D, Moris S M and Coles H J, 2006. Photonics and lasing in liquid crystals. Mater. Today. 9: $36-42$.

49. Coles H and Morris S, 2010. Liquid-crystal lasers. Nature Photonics. 4: 676-685.

50. Palto S P, 2006. Lasing in liquid crystal thin films. JETP. 103: 472-479.

51. Kopp V I, Zhang Zh-Q and Genack A Z, 2003. Lasing in chiral photonic structures. Progr. Quant. Electron. 27: 369416. 
52. Fuh A Y-G, Lin T-H, Liu J-H and Wu F-C, 2004. Lasing in chiral photonic liquid crystals and associated frequency tuning. Opt. Express. 12: 1857-1863.

53. Palto S P, Shtykov N M, Umansky B A, Barnik M I and Blinov L M, 2006. General properties of lasing effect in cholesteric liquid crystals. Opto-Electron. Rev. 14: 323-328.

54. Lee C-R, Lin S-H, Yeh H-C, Ji T-D, Lin K-L, Mo T-S, Kuo C-T, Lo K-Y, Chang S-H, Fuh A Y-G and Huang S-Y, 2009. Color cone lasing emission in a dye-doped cholesteric liquid crystal with a single pitch. Opt. Express. 17: 12910-12921.

55. Lee C-R, Lin S-H, Yeh H-C and Ji T-D, 2009. Band-tunable color cone lasing emission based on dye-doped cholesteric liquid crystals with various pitches and a pitch gradient. Opt. Express. 17: 22616-22623.

56. Lee $\mathrm{C}-\mathrm{R}$, Lin $\mathrm{S}-\mathrm{H}, \mathrm{Ku} \mathrm{H}-\mathrm{S}$, Liu J-H, Yang $\mathrm{P}-\mathrm{C}$, Huang S-Y, Yeh H-C, Ji T-D and Lin C-H, 2010. Spatially band-tunable color-cone lasing emission in a dye-doped cholesteric liquid crystal with a photoisomerizable chiral dopant. Opt. Lett. 35: 1398-1400.

57. Lin S-H and Lee C-R, 2011. Novel dye-doped cholesteric liquid crystal cone lasers with various birefringences and associated tunabilities of lasing feature and performance. Opt. Express. 19: 18199-18206.

58. Palto S P, Shtykov N M, Umanskii B A and Barnik M I, 2012. Multiwave out-of-normal band-edge lasing in cholesteric liquid crystals. J. Appl. Phys. 112: 013105-8.

59. Penninck L, Beeckman J, De Visschere P and Neyts K, 2012. Light emission from dye-doped cholesteric liquid crystals at oblique angles: Simulation and experiment. Phys. Rev. E. 85: 041702-7.

60. Blinov L M, Cipparrone G, Pagliusi P, Lazarev V V and Palto S P, 2006. Mirrorless lasing from nematic liquid crystals in the plane waveguide geometry without refractive index or gain modulation. Appl. Phys. Lett. 89: 0311114-3.

61. Blinov L M, Cipparrone G, Mazzulla A, Pagliusi P, Lazarev V V and Palto S P, 2008. Quasi-in-plane leaky modes in lasing cholesteric liquid crystal cells. J. Appl. Phys. 104: 103115-7.

62. Yoshida H, Inoue Y, Isomura T, Matsuhisa Y, Fujii A and Ozakib M, 2009. Position sensitive, continuous wavelength tunable laser based on photopolymerizable cholesteric liquid crystals with an in-plane helix alignment. Appl. Phys. Lett. 94: 093306-3.

63. Morris S M, Ford A D, Pivnenko M N and Coles H J, 2005. The effects of reorientation on the emission properties of a photonic band edge liquid crystal laser. J Opt. A: Pure Appl. Opt. 7: 215-223.

64. Cao W, Palffy-Muhoray P, Taheri B, Marino A and Abbate G, 2005. Lasing thresholds of cholesteric liquid crystals lasers. Mol. Cryst. Liq. Cryst. 429: 101-110.

65. Morris S M, Ford A D, Gillespie C, M N Pivnenko, Hadeler O and Coles H J, 2006. The emission characteristics of liquid-crystal lasers. J. SID. 14: 565-573.

66. Woon K L, O'Neill M, Richards G J, Aldred M P and Kelly S M, 2005. Stokes parameter studies of spontaneous emission from chiral nematic liquid crystals as a one-dimensional photonic stopband crystal: Experiment and theory. Phys. Rev. E. 71: 041706-8.

67. Watanabe Y, Uchimura M, Araoka F, Konishi G-I, Watanabe J and Takezoe H, 2009. Extremely low threshold in a pyrene-doped distributed feedback cholesteric liquid crystal laser. Appl. Phys. Express. 2: 102501-3.

68. Dolgaleva K, Wei S K H, Lukishova S G, Chen S H, Schwertz K and Boyd R W, 2008. Enhanced laser performance of cholesteric liquid crystals doped with oligofluorene dye. J. Opt. Soc. Amer. B. 25:1496-1504.

69. Förster T, 1959. Transfer mechanisms of electronic excitation. Disc. Faraday Soc. 27: 717 
70. Berggren M, Dodabalapur A, Slusher R E and Bao Z, 1997. Light amplification in organic thin films using cascade energy transfer. Nature. 389: 466-469.

71. Alvarez E, He M, Munoz A F, Palffy-Muhoray P, Serak S V, Taheri B and Twieg R, 2001. Mirrorless lasing and energy transfer in cholesteric liquid crystals doped with laser dyes. Mol. Cryst. Liq. Cryst. 369: 75-82.

72. Chambers M, Fox M and Grell M, 2002. Lasing from a Förster transfer fluorescent dye couple dissolved in a chiral nematic liquid crystal. Adv. Func. Mater. 12: 808-810.

73. Sonoyama K, Takanishi Y, Ishikawa K and Takezoe H, 2008. Lowering threshold by energy transfer between two dyes in cholesteric liquid crystal distributed feedback lasers. Appl. Phys. Express. 1: 032002-3.

74. Morris S M, Ford A D, Pivnenko M N and Coles H J, 2005. Enhanced emission from liquid-crystal lasers. J. Appl. Phys. 97: 023103-9.

75. Morris S M, Ford A D, Pivnenko M N, Hadeler O and Coles H J, 2006. Correlations between the performance characteristics of a liquid crystal laser and the macroscopic material properties. Phys. Rev. E. 74: 061709-5.

76. Ford A D, Morris S M, Pivnenko M N, Gillespie C O and Coles H J, 2007. Emission characteristics of a homologous series of bimesogenic liquid-crystal lasers. Phys. Rev. E. 76: 051703-9.

77. Chee M G, Song M H, Kim D, Takezoe H and Chung I J, 2007. Lowering lasing threshold in chiral nematic liquid crystal structure with different anisotropies. Jpn. J. Appl. Phys. 18: L437-L439.

78. Huang $\mathrm{Y}$, Zhou $\mathrm{Y}$, Hong Q, Rapaport A, Bass M and $\mathrm{Wu}$ S-T, 2006. Incident angle and polarization effects on the dye-doped cholesteric liquid crystal laser. Opt. Commun. 261: 91-96.

79. Huang Y, Lin T-H, Zhou Y and Wu S-T, 2006. Enhancing the laser power by stacking multiple dye-doped chiral polymer films. Opt. Express. 14: 11299-11303.

80. Shtykov N M, Barnik M I, Blinov L M, Umanskii B A and Palto S P, 2007. Amplification of the emission of a liquid-crystal microlaser by means of a uniform liquid-crystal layer. JETP Lett. 85: 602-604.

81. Wang Y, Manabe T, Takanishi Y, Ishikawa K, Shao G, Orita A, Otera J and Takezoe H, 2007. Dependence of lasing threshold power on excitation wavelength in dye-doped cholesteric liquid crystals. Opt. Commun. 280: 408-411.

82. Mowatt C, Morris S M, Song M H, Wilkinson T D, Friend R H and Coles H J, 2010. Comparison of the performance of photonic band-edge liquid crystal lasers using different dyes as the gain medium. J. Appl. Phys. 107: 043101-9.

83. Chanishvili A, Chilatya G, Petriashvili G, Barberi R, Bartolino R, Cipparone G, Mazzulla A and Oriol L, 2004. Lasing in dye-doped cholesteric liquid crystal: two new tuning strategies. Adv. Mater. 16: 791-795.

84. Furumi S, Yokoyama S, Otomo A and Mashiko S, 2004. Phototunable photonic bandgap in a chiral liquid crystal laser device. Appl. Phys. Lett. 84: 2491-2493.

85. Bobrovsky A Yu, Boiko N I, Shibaev V P and Wendorf J H, 2003. Cholesteric mixtures with photochemically tunable circularly polarized fluorescence. Adv. Mater. 15: 282-287.

86. Huang Y, Chen L-P, Doyle Ch, Zhou Y and Wu S-T, 2006. Spatially tunable laser emission in dye-doped cholesteric polymer films. Appl. Phys. Lett. 88: 011107.

87. Sonoyama K, Takanishi Y, Ishikawa K and Takezoe H, 2007. Position-sensitive cholesteric liquid crystal dye laser covering a full visible range. Jpn. J. Appl. Phys. 46: L874-L876.

88. Wang $\mathrm{Ch}-\mathrm{T}$ and Lin $\mathrm{T}-\mathrm{H}, 2008$. Multi-wavelength laser emission in dye-doped photonic liquid

Ukr. J. Phys. Opt. 2014, Volume 15, Issue 2 
crystals. Opt. Express. 16: 18334-18339.

89. Funamoto K, Ozaki M and Yoshino K, 2003. Discontinuous shift of lasing wavelength with temperature in cholesteric liquid crystal. Jpn. J. Appl. Phys. 42: L523-L525.

90. Morris S M, Ford A D and Coles H J, 2009. Removing the discontinuous shifts in emission wavelength of a chiral nematic liquid crystal laser. J. Appl. Phys. 106: 023112-4.

91. De Gennes P G, 1968. Calcul de la distorsion d'une structure cholesterique par un champ magnetique. Solid State Commun. 6: 163-165.

92. Kasano M, Ozaki M, Yoshino K, Ganzke D and Haase W, 2003. Electrically tunable waveguide laser based on ferroelectric liquid crystal. Appl. Phys. Lett. 82: 4026-4028.

93. Shirota K, Sun H-Bo and Kawata S, 2004. Two-photon lasing of dye-doped photonic crystal lasers. Appl. Phys. Lett. 84: 1632-1634.

94. Chanishvili A, Chilaya G, Petriashvili G, Barberi R, Bartolino R, Cipparrone G and Mazzulla A, 2004. Laser emission from a dye-doped cholesteric liquid crystal pumped by another cholesteric liquid crystal laser. Appl. Phys. Lett. 85: 3378-3380.

95. Shibaev P V, Tang K, Genack A Z, Kopp V and Green M M, 2002. Lasing from a stiff chain polymeric lyotropic cholesteric liquid crystal. Macromolecules. 35: 3022-3025.

96. Schmidtke J, Stille W, Finkelman H and Kim S T, 2002. Laser emission in a dye doped cholesteric polymer network. Adv. Mater. 14: 746-749.

97. Finkelmann H, Kim S T, Munoz A, Palffy-Muhoray P and Taheri B, 2001. Tunable mirrorless lasing in cholesteric liquid crystalline elastomer. Adv. Mater. 13: 1069-1072.

98. Matsui T, Ozaki R, Funamoto K, Ozaki M and Yoshino K, 2002. Flexible mirrorless laser based on a free-standing film of photopolymerized cholesteric liquid crystal. Appl. Phys Lett. 81: 3741-3743.

99. Araoka F, Shin K-Ch, Takanishi Y, Ishikawa K, Takezoe H, Zhu Zh and Swager T M, 2003. How doping a cholesteric liquid crystal with polymeric dye improves an order parameter and makes possible low threshold lasing. J. Appl. Phys. 94: 279-283.

100. Yablonovitch E, Gmitter T J, Meade R D, Rappe A M, Brommer K D and Joannopoulos J D, 1991. Donor and acceptor modes in photonic band structure. Phys. Rev. Lett. 67: 3380-3383.

101. Stoytchev M and Genack A Z, 1997. Microwave transmission through a periodic three-dimensional metal-wire network containing random scatterers. Phys. Rev. B. 55: R8617-8621.

102. Chabanov A A, Stoytchev M and Genack A Z, 2000. Statistical signatures of photon localization. Nature. 404: 850-853.

103. Yang Y-Ch, Kee Ch-S, Kim J-E and Park H Y, 1999. Photonic defect modes of cholesteric liquid crystals. Phys. Rev. E. 60: 6852-6854.

104.Barnik M I, Blinov L M, Lazarev V V, Palto S P, Umanskii B A and Shtykov N M, 2008. Lasing from photonic structure: Cholesteric-voltage controlled nematic-cholesteric liquid crystal. J. Appl. Phys. 103: 123113-7.

105.Song M H, Park B, Shin K-Ch, Ohta T, Tsunoda Y, Hoshi H, Takanishi Y, Ishikava K, Watanabe J, Nishimura S, Toyooka T, Zhu Zh, Swager T M and Takezoe H, 2004. Effect of phase retardation on defect mode lasing in polymeric cholesteric liquid crystals. Adv. Mater. 16: 779-783.

106. Song M H, Park B, Toyooka T, Chung I J, Takanishi Y, Ishikava K and Takezoe H, 2006. Electrotunable non-reciprocal laser emission from a liquid-crystal photonic device. Adv. Func. Mater. 16: 1793-1798.

107.Kopp V I and Genack A Z, 2002. Twist defect in chiral photonic structures. Phys. Rev. Lett. 89: 033901-4.

108. Schmidtke J, Stille W and Finkelman H, 2003. Defect mode emission of a dye doped cholesteric 
polymer network. Phys. Rev. Lett. 90: 083902-4.

109. Schmidtke J and Stille W, 2003. Photonic defect modes of cholesteric liquid crystal films. Eur. Phys. J. E. 12: 553-564.

110. Becchi M, Ponti S, Reyes J A and Oldano C, 2004. Defect modes in helical photonic crystals: An analytic approach. Phys. Rev. B. 70: 033103-4.

111. Matsui T, Ozaki M and Yoshino K, 2004. Tunable photonic defect modes in a cholesteric liquid crystal induced by optical deformation of helix. Phys. Rev. E. 69: 061715-4.

112. Takanishi Y, Tomoe N, Ha N Y, Toyooka T, Nishimura S, Ishikava K and Takezoe H, 2007. Defect-mode lasing from a three-layered helical cholesteric liquid crystal structure. Jpn. J. Appl. Phys. 46: 3510-3513.

113. Song M H, Ha Y, Amemiya K, Park B, Takanishi Y, Ishikava K, Wu J W, Nishimura S, Toyooka T and Takezoe H, 2006. Defect-mode lasing with lowered threshold in three-layered hetero-cholesteric liquid-crystal structure. Adv. Mater. 18: 193-197.

114. Takanishi Y, Ohtsuka Y, Suzaki G, Nishimura S and Takezoe H, 2010. Low threshold lasing from dye-doped cholesteric liquid crystal multi-layered structures. Opt. Express. 18: 12909-12914.

115. Ha N Y, Takanishi Y and Takezoe H, 2007. Simultaneous RGB reflections from single-pitched cholesteric liquid crystal films with Fibonaccian defects. Opt. Express. 15: 1024-1029.

116. Ha N Y, Ohtsuka Y, Jeong S M, Nishimura S, Suzaki G, Takanishi Y, Ishikava K and Takezoe H, 2008. Fabrication of a simultaneous red-green-blue reflector using single-pitched cholesteric liquid crystals. Nature. 7: 43-47.

117. Gellermann W, Kohmoto M, Sutherland B and Taylor P C, 1994. Localization of light waves in Fibonacci dielectric multilayers. Phys. Rev. Lett. 72: 633636.

118. Negro L D, Stolfi M, Yi Y, Michel J, Duan X, Kimerling L C, Le Blanc J and Haavisto J, 2004. Photon band gap properties and omnidirectional reflectance in $\mathrm{Si} / \mathrm{SiO} 2$ Thue-Morse quasicrystals. Appl. Phys. Lett. 84: 51865188.

119. Vasconcelos M S and Albuquerque E L, 1999. Transmission fingerprints in quasiperiodic dielectric multilayers. Phys. Rev. B. 59: 11128-11131.

120. Ganic K, Gan X, Gu M, Hain M, Somalingam S, Stankovic S and Tschudi T, 2002. Generation of doughnut laser beams by use of a liquid-crystal cell with a conversion efficiency near $100 \%$. Opt. Lett. 27: 13511353.

121. Voloschenko D and Lavrentovich O D, 2000. Optical vortices generated by dislocations in a cholesteric liquid crystal. Opt. Lett. 26: 317-319.

122.Matsko A B, Savchenkov A A, Strekalov D, Ilchenko V S and Maleki L, 2005. Review of applications of whispering-gallery mode resonators in photonics and nonlinear optics. 4, IPN Progress Rep. 15: 42-162.

123.Lord Rayleigh, 1910. The problem of whispering gallery. Philos. Mag. 20: 1001-1004.

124. Vahala K J, 2003. Optical microcavities. Nature. 424: 841-846.

125. Chiasera A, Dumeige Y, F'ron P, Ferrari M, Jestin Y, Conti G N, Pelli S, Soria S and Righini G C, 2010. Spherical whispering-gallery-mode microresonators. Laser \& Photon. Rev. 4: 457482.

126. Collot L, Lef" vre-Seguin V, Brune M, Raimond J M and Haroche S, 1993. Very high-Q whispering-gallery mode resonances observed on fused silica microspheres. Europhys. Lett. 23: 327-334.

127. Sandoghdar V, Treussart F, Hare J, Lef vre-Seguin V, Raimond J M and Haroche S, 1996. Very low threshold whispering-gallery-mode microsphere laser. Phys. Rev. A. 54: R1777-R1780.

128. Garret C G B, Kaiser W and Bond W L, 1961. Stimulated emission into optical whispering modes of spheres. Phys. Rev. 124: 1807-1809. 
129. Cai M, Painter O and Vahala K J, 2000. Fiber-coupled microsphere laser. Opt. Lett. 25: $1430-1432$.

130. Yang L and Vahala K J, 2003. Gain functionalization of silica microresonators. Opt. Lett. 28: 592-594.

131.Kuwata-Gonokami M and Takeda K, 1998. Polymer whispering gallery mode lasers. Opt. Mater. 9: 12-17.

132. Cha J N, Bartl M H, Wong M S, Popitsch A, Deming T J and Stucky G D, 2003. Microcavity lasing from block peptide hierarchically assembled quantum dot spherical resonators. Nano Lett. 3: 907-911.

133. Yamaguchi K, Niimi T, Haraguchi M, Ookamoto T and Fukui M, 2006. Fabrication and optical evaluation of silica microsphere coated with J-aggregates. Jpn. J. Appl. Phys. 45: 6750-6753.

134. Nöckel J U, Stone A D, Chen G, Grossman H L and Chang R K, 1996. Directional emission from asymmetric resonant cavities. Opt. Lett. 21: 1609-1611.

135. Gmachl C, Capasso F, Narimanov E E, Nöckel J U, Stone A D, Faist J, Sivco D L and Cho A Y, 1998. High-power directional emission from microlasers with chaotic resonators. Science. 280: 1556-1564.

136. Lacey S and Wang H, 2001. Directional emission from whispering-gallery modes in deformed fused-silica microspheres Opt. Lett. 26: 1943-1945.

137. Wang Q J, Yan C, Yu N, Unterhinninghofen J, Wiersig J, Pflügl C, Diehl L, Edamura T, Yamanishi M, Kan H and Capasso F, 2010. Whispering-gallery mode resonators for highly unidirectional laser action. Proc. Nat. Acad. Sci. U.S.A. 107: 22407-22412.

138. Wiersig J, Unterhinninghofen J, Song Q, Cao H, Hentschel M and Shinohara S, 2011. Review on unidirectional light emission from ultralow-loss modes in deformed microdisks. Trends in Nano- and Micro-Cavities. 2011: 109-152.

139.Psaltis D, Quake S R and Yang C, 2006. Developing optofluidic technology through the fusion of microfluidics and optics. Nature. 442: 381-386.

140.Kou Q, Yesilyurt I and Chen Y, 2006. Collinear dual-color laser emission from a microfluidic dye laser. Appl. Phys. Lett. 88: 091101-3.

141.Li Z, Zhang Z, Scherer A and Psaltis D, 2006. Mechanically tunable optofluidic distributed feedback dye laser. Opt. Express. 14: 10494-10499.

142. Gersborg-Hansen M and Kristensen A, 2007. Tunability of optofluidic distributed feedback dye lasers. Opt. Express. 15: 137-142.

143. Song W, Vasdekis A E, Li Z and Psaltis D, 2009. Optofluidic evanescent dye laser based on a distributed feedback circular grating. Appl. Phys. Lett. 94: 161110-3.

144. Aubry G, Kou Q, Soto-Velasco J, Wang C, Meance S, He J J and Haghiri-Gosnet A M, 2011. A multicolor microfluidic droplet dye laser with single mode emission. Appl. Phys. Lett. 98: 111111-3.

145.Humar M and Muševič I, 2011. Surfactant sensing based on whispering-gallery-mode lasing in liquid-crystal microdroplets. Opt. Express. 19: 19836-19844.

146. Gottardo S, Cavalieri S, Yaroshchuk O and Wiersma D S, 2004. Quasi-two-dimensional diffusive random laser action. Phys. Rev. Lett. 93: 263901-4.

147.Liu Y J, Suna X W, Elim H I and Ji W, 2006. Gain narrowing and random lasing from dye-doped polymer-dispersed liquid crystals with nanoscale liquid crystal droplets. Appl. Phys. Lett. 89: 011111-3.

148. Humar M and Muševič I, 2010. 3D microlasers from self-assembled cholesteric liquid-crystal microdroplets. Opt. Express. 18: 26995-27003.

149. Kurik M V and Lavrentovich O D, 1982. Negative-positive monopole transitions in cholesteric 
liquid crystals. JETP Lett. 35: 444-447.

150. Nastishin Yu A, Kléman M, Malthête J and Nguyen H T, 2001. Identification of a TGBA liquid crystal phase via its defects. Eur. Phys. J. E. 5: 353-357.

151.Kléman M, Nastishin Yu A and Malthête J, 2002. Defects in a TGBA phase: A theoretical approach. Eur. Phys. J. E. 8: 67-78.

152.Lin J-D, Hsieh M-H, Wei G-J, Mo T-S, Huang S-Y and Lee C-R, 2013. Optically tunable/switchable omnidirectionally spherical microlaser based on a dye-doped cholesteric liquid crystal microdroplet with an azo-chiral dopant. Opt. Express. 21: 15765-15776.

Nastishin Yu. A. and Dudok T. H., 2014. Optically pumped mirrorless lasing. A Review. Part II. Lasing in photonic crystals and microcavities. Ukr. J. Phys. Opt. 15: 47 - 67.

Анотація. Ця стаття є другою частиною огляду про бездзеркальну лазерну генерацію з оптичним нагнітанням. Розгляд випадкової лазерної генераиії, представлений у першій частині огляду [Nastishin Yu A and Dudok T H, 2013. Ukr. J. Phys. Opt.], тут продовжено аналізом літератури з генераиї фотонними структурами, зокрема холестеричними рідкими кристалами з домішками барвника і мікрорезонаторами з включенням мікрокрапель рідких кристалів. 